\title{
Bañados-Silk-West effect with nongeodesic particles: extremal horizons
}

\author{
I. V. Tanatarov ${ }^{1,2, *}$ and O. B. Zaslavskii ${ }^{2, \dagger}$ \\ ${ }^{1}$ Kharkov Institute of Physics and Technology, \\ 1 Akademicheskaya, Kharkov 61108, Ukraine \\ ${ }^{2}$ Department of Physics and Technology, \\ Kharkov V.N. Karazin National University, \\ 4 Svoboda Square, Kharkov 61022, Ukraine
}

The Bañados-Silk-West (BSW) effect consists in the possibility to obtain arbitrarily large energy $E_{c . m}$. in the centre of mass frame of two colliding particles near the black hole horizon. One of the common beliefs was that the action of force on these particles (say, due to gravitational radiation) should necessarily restrict the growth of $E_{c . m .}$. We consider extremal horizons and develop a model-independent approach and analyze the conditions for the force to preserve or kill the effect, using the frames attached both to observers orbiting the black hole and to ones crossing the horizon. We argue that the aforementioned expectations are not confirmed. Under rather general assumptions, the BSW effect survives. For equatorial motion it is only required that in the proper frame the radial component of the force be finite, while the azimuthal one tend to zero not too slowly. If the latter condition is violated, we evaluate $E_{c . m .}$, which becomes indeed restricted but remains very large for small forces.

PACS numbers: 04.70.Bw, 97.60.Lf

Keywords: BSW effect, backreaction force

*Electronic address: igor.tanatarov@gmail.com

${ }^{\dagger}$ Electronic address: zaslav@ukr.net 


\section{Contents}

$\begin{array}{ll}\text { I. Introduction } & 3\end{array}$

II. Particles' kinematics near extremal horizons 5

A. A particle in axially symmetric metric 5

B. Two particles' collision near horizon 6

C. Usual and critical particles near extremal horizons $\quad 7$

1. Example: the Kerr metric 9

D. The BSW effect 9

E. Generalization: usual, critical and sub-critical particles 10

III. Dynamics 12

A. OZAMO and FZAMO frames 12

B. Acceleration in different frames 16

C. Energy and angular momentum 16

D. Dynamic restrictions on a particle's velocity 17

$\begin{array}{ll}\text { E. Usual particles } & 18\end{array}$

F. Critical particles $\quad 19$

IV. Example: the Reissner-Nordström metric 21

A. Static observers 22

B. Falling observers 23

V. BSW effect under finite forces: equatorial motion 24

A. Motion in equatorial plane 24

B. Acceleration in proper frame 26

1. Usual particles 26

2. Sub-critical particles $\quad 27$

3. Critical particles 27

C. Example: azimuthal dissipative force 28

1. Tuning a critical particle 28

2. Other realizations of critical trajectories 30

VI. Energy bounds in collisions with near-critical particles 31

VII. Kinematic restrictions on critical particles and two types of the BSW effect 32

$\begin{array}{ll}\text { VIII. Conclusion } & 34\end{array}$

References $\quad 35$ 


\section{INTRODUCTION}

Recently, an interesting effect was discovered by Bañados, Silk and West [1], called usually the BSW effect after the names of the authors: if two particles collide near the black hole horizon, the energy $E_{c . m}$. in their centre of mass frame can grow indefinitely large, provided the parameters of one of the particles are fine-tuned. Immediately after this observation, several considerations of theoretical nature were brought forward suggesting that there must be restrictions that would prevent the realization of this effect. One of the basic objections is connected with the force of gravitational radiation acting on particles. It was pushed forward in [2] and is mentioned from time to time in consequent works starting from [3]. There are also other similar effects which seem to restrict the divergence of $E_{c . m}$. - say, synchrotron radiation by charged particles near black holes [4].

Meanwhile, the influence of the force of gravitational radiation (or any other force) on the BSW effect is not so obvious. First of all, the BSW effect is prepared from two main ingredients - the presence of the horizon and the presence of special "critical" trajectories, (see below). It was shown in [5], with minimal assumptions, that even for neutral particles and nongeodesic motion, such trajectories do exist. Therefore, the question is whether or not the force destroys these trajectories. If this happens, the BSW effect is restricted. However, for a weak force, one can expect a large bound on $E_{c . m .}$. For instance, the analysis of particle's motion on the innermost stable orbit near the Kerr black hole with gravitational radiation taken into account showed that $E_{c . m}$. can be far beyond the Planck energy for collision of dark matter particles near a stellar mass near-extremal black hole [6]. The analysis suggested in [6], however, concerns special (although important for astrophysics) cases: it applies to near-extremal Kerr black holes when fine-tuning required for the BSW effect is realized on circular orbits. It also remains incomplete since not all factors responsible for the self-force are taken into account. Meanwhile, it is of interest to elucidate the issue under discussion in a model-independent way.

In this paper we develop such a general approach and analyze the BSW effect under the influence of a generic force near the horizon of a generic axially symmetric stationary "dirty" black hole (i.e. a black hole that is surrounded by matter, so its metric may deviate from the Kerr one). Here we only consider the case of an extremal horizon of a maximally rotating black hole. The approach used is applicable, with minimal modifications, to static 
or charged black holes, as shown explicitly for the case of Reissner-Nordström metric.

We consider the conditions the force should satisfy for the effect to be either preserved in some form or not. The analysis is made in terms of tetrad components of the corresponding quantities in the frames attached both to an observer orbiting the black hole, and the one crossing the horizon. The nature of the force itself is not specified, we only assume that its tetrad components in the particle's proper frame are finite and restrict our consideration to equatorial motion. We show that the BSW effect survives any force that satisfies the following assumptions: (i) it remains finite near the horizon, and (ii) its azimuthal component tends to zero fast enough (more detailed definition is given below). In case the above condition is not satisfied, e.g. the azimuthal force does not vanish in the horizon limit, the weaker version of the effect is realized whenever the acceleration's amplitude is small enough (as should be for e.g. radiation reaction). For the latter case, we find generic bounds on $E_{\text {c.m. }}$.

It is worth stressing that the BSW effect reveals itself not only for extremal black holes, but also for nonextremal ones. The mechanism in the latter case, however, is generally different, as it requires multiple scattering, which for extremal black holes is not necessary [7] (see also [8]). Correspondingly, we postpone consideration of the BSW effect with a force near nonextremal horizons and, in the present paper, restrict ourselves to the extremal case. The effect for near-extremal horizons, considered in [6], occupies an intermediate position between the two. This problem contains some subtleties on its own related to the properties of near-circular orbits and in the general setting also needs separate treatment.

There are two aspects of the BSW effect - the behavior of $E_{c . m}$. near the horizon and the properties of energies of the collision outcome measured at infinity. The typical energies at infinity are quite modest even in the absence of force [9-11], so taking the force into account can only change them slightly. It is the first aspect which is nontrivial and is being discussed in the present paper.

The paper is organized as follows. In Sec. II, we consider classification of particles relevant for the BSW effect and discuss novel features that the force brings into the system. In Sec. III, we consider behavior of acceleration near the horizon in different frames (attached to an observer orbiting the black hole or to one crossing the horizon). In Sec. IV, we illustrate general relationships using the Reissner-Nordström metric as an example. In Sec. V, we consider generic motion in the equatorial plane under the action of finite forces and derive 
conditions on the force that allow or forbid critical trajectories. In Sec. VI, we estimate the bounds on $E_{c . m}$. for the case when the force is least favourable for the effect but small. In Sec. VII, we discuss pure kinematic restrictions on particle's trajectories (valid even in the absence of force) which can influence the properties of the BSW effect. Sec. VIII is devoted to conclusion.

\section{PARTICLES' KINEMATICS NEAR EXTREMAL HORIZONS}

\section{A. A particle in axially symmetric metric}

We consider the axially symmetric stationary metric written (at least in the vicinity of the horizon) in coordinates which are obtained from the Gaussian normal ones by replacing the distance to the horizon $n$ with the radial coordinate $r$, defined so that ${ }^{1} A(r) \sim N^{2}$ in the horizon limit, where $N^{2} \rightarrow 0$ (hereafter $c=1$ ):

$$
d s^{2}=-N^{2} d t^{2}+g_{\phi}(d \phi-\omega d t)^{2}+\frac{d r^{2}}{A}+g_{z} d z^{2} .
$$

Let there be some arbitrary, not necessarily geodesic, particle of mass $m$, four-velocity $u^{\mu}$ and four-momentum

$$
p^{\mu}=m u^{\mu} .
$$

It is convenient to represent the four-velocity, both with upper and lower indices, by the components of its four-momentum in the following way:

$$
\begin{aligned}
& u^{\mu}=\frac{1}{m}\left(\frac{X}{N^{2}}, \frac{L}{g_{\phi}}+\frac{\omega X}{N^{2}}, p^{r}, p^{z}\right) ; \\
& u_{\mu}=\frac{1}{m}\left(-E, L, \frac{1}{A} p^{r}, g_{z} p^{z}\right),
\end{aligned}
$$

where $E=-m u_{0}$ is energy, $L=m u_{\phi}$ angular momentum and

$$
X=E-\omega L .
$$

\footnotetext{
${ }^{1}$ Such as the quasiglobal coordinate of [12], ch.3.
} 
Due to forward in time condition, $X$ is always positive.

For a free particle on a geodesic trajectory the energy $E$ and angular momentum $L$ are conserved; eqs. (2), (3) are nothing but the equations of motion with given fixed values of $E$ and $L$. In the general case, $E$ and $L$ are not conserved and together with $u^{z}$ should be treated as functions of the particle's proper time. Nonetheless, we still write the components of the four-velocity in the same form (2), (3) which can be considered simply as useful parametrization.

The normalization condition $u^{\mu} u_{\mu}=-1$ can be written as

$$
\frac{1}{A}\left(p^{r}\right)^{2}+g_{z}\left(p^{z}\right)^{2}=\frac{X^{2}}{N^{2}}-\frac{L^{2}}{g_{\phi}}-m^{2} .
$$

Then $p^{r}$ is expressed through the three independent parameters $E, L$ and $u^{z}$ :

$$
p^{r}= \pm \frac{\sqrt{A}}{N} Z
$$

where

$$
Z^{2}=X^{2}-N^{2}\left[\frac{L^{2}}{g_{\phi}}+g_{z}\left(p^{z}\right)^{2}+m^{2}\right]
$$

The formulas in this section are applicable also to massless particles, with the only difference that one has to set $m=0$. The four-momentum then, in the appropriate parametrization of the worldline, is related to the wave vector $k^{\mu}$ as $p^{\mu}=\hbar k^{\mu}$.

\section{B. Two particles' collision near horizon}

The energy $E_{i \text { c.m. }}$ of a particle $i$ with four-momentum $p_{i}^{\mu}$ in its center of mass (c.m.) frame is simply its rest mass, i.e. the norm of its four-momentum:

$$
E_{i c . m .}^{2}=m_{i}^{2}=-p_{i}^{\mu} p_{i \mu}
$$


Likewise, for two particles with masses $m_{1}$ and $m_{2}$ and four-velocities $u_{1}^{\mu}$ and $u_{2}^{\mu}$ the center of mass energy $E_{c . m}$. at the collision event is the norm of their total four-momentum

$$
E_{c . m .}^{2}=-\left(p_{1}^{\mu}+p_{2}^{\mu}\right)\left(p_{1 \mu}+p_{2 \mu}\right)=m_{1}^{2}+m_{2}^{2}+2 m_{1} m_{2} \gamma_{c . m .},
$$

where

$$
\gamma_{c . m .}=-u_{1 \mu} u_{2}^{\mu}
$$

is the relative Lorentz factor.

The contraction can be written as

$$
m_{1} m_{2} \gamma_{c . m .}=\frac{X_{1} X_{2}-Z_{1} Z_{2}}{N^{2}}-\frac{L_{1} L_{2}}{g_{\phi}}-g_{z} p_{1}^{z} p_{2}^{z} .
$$

For a collision of a massive particle of mass $m$ and a photon one obtains that

$$
E_{c . m .}^{2}=m^{2}+2 m \hbar \omega_{\text {det }},
$$

where $\omega_{\text {det }}=-k_{\mu} u^{\mu}$ is the photon's frequency as detected in the frame of this massive particle.

\section{Usual and critical particles near extremal horizons}

Consider a particle in the vicinity of a regular extremal horizon, for which [13]

$$
\begin{aligned}
N^{2}(r) & \sim\left(r-r_{H}\right)^{2}, \\
\omega(r) & =\omega_{H}-\omega_{1}(z) N+O\left(N^{2}\right), \quad \omega_{H}=\text { const },
\end{aligned}
$$

where $r=r_{H}$ is the horizon. The regularity of the horizon implies [13] that, in particular, $\omega_{H}$ is a constant, and that other metric functions can also be expanded into series by $\left(r-r_{H}\right)$ with positive powers. Note also the sign by $\omega_{1}$, defined so for consistency with earlier works.

From the normalization condition (5) then a particle's four-velocity components can always be presented as series by $N$ (though they can diverge at the horizon). Assuming $L$ 
and $E$ are finite,

$$
E=E_{H}+E_{1} N+O\left(N^{2}\right), \quad L=L_{H}+L_{1} N+O\left(N^{2}\right),
$$

and then

$$
\begin{aligned}
X=X_{H} & +O(N), \\
& X_{H}=E_{H}-\omega_{H} L_{H} .
\end{aligned}
$$

For a usual (generic) particle $X_{H} \neq 0$. The normalization (5) then implies that

$$
u^{r}=O(1)
$$

$u^{z}=O(1 / N)$, so the particle reaches the horizon in finite proper time $\tau \sim \int d r<\infty$.

However, there are also worldlines of particles with angular momentum fine-tuned to energy in such a way that $X_{H}=0$, so that

$$
X=O(N)
$$

Such particles are called critical.

For critical particles the right hand side of normalization condition(5) is bounded, and as the left hand side there is a sum of squares, we obtain

$$
u^{r}=O(N), \quad u^{z}=O(1)
$$

Then the equation of radial motion in the main order by $N$ is

$$
\frac{d r}{d \tau}=-\frac{r-r_{H}}{\tau_{0}}
$$


where $\tau_{0}$ is a constant for motion in equatorial plane ${ }^{2}$; its solution is

$$
r-r_{H}=r_{0} e^{-\tau / \tau_{0}}
$$

and the proper time of reaching the horizon diverges as $\ln \left(r-r_{H}\right)$. In case $u^{r}$ is of higher order that $N$, the divergence is stronger (i.e. if $u^{r} \sim\left(r-r_{H}\right)^{2}$, then $\tau$ diverges as $\left(r-r_{H}\right)^{-1}$ ).

\section{Example: the Kerr metric}

It is instructive to look at the critical trajectory for the Kerr metric. Let us restrict ourselves to equatorial motion $\theta=\frac{\pi}{2}$. Then, the metric coefficients near the horizon of the extremal Kerr black hole read

$$
\begin{aligned}
& N \approx \frac{r-r_{H}}{2 r_{H}}, \quad \sqrt{A} \approx \frac{r-r_{H}}{r_{H}}, \quad\left(g_{\phi}\right)_{H}=4 r_{H}^{2}, \\
& \omega_{H}=\frac{1}{2 r_{H}}, \quad \omega-\omega_{H} \approx-\frac{r-r_{H}}{2 r_{H}^{2}} .
\end{aligned}
$$

Then, it follows from eqs. (6), (7) that the trajectory of the particle with $E=\omega_{H} L$ has exactly the form (21) with

$$
\tau_{0}=\frac{r_{H}}{\sqrt{3 \frac{E^{2}}{m^{2}}-1}}
$$

\section{The BSW effect}

Consider the collision of two particles. For a usual (generic) particle, assuming $E, L$ and $u^{z}$ are finite ${ }^{3}$,

$$
X=X_{H}+O(N), \quad Z=X+O\left(N^{2}\right) .
$$

Then the relative Lorentz factor at the collision event of two usual particles is

$$
m_{1} m_{2} \gamma_{c . m .}=\frac{X_{1} X_{2}-Z_{1} Z_{2}}{N^{2}}+O(1)=O(1)
$$

\footnotetext{
${ }^{2}$ In general, the coordinate $z$ can oscillate between some limiting values, see [16] for the Kerr metric and [21] for discussion of a more general case.

${ }^{3}$ Those are natural assumptions, but for justification see the section on dynamics below.
} 
However, for a critical particle

$$
X=X_{N} N+O\left(N^{2}\right), \quad Z=Z_{N} N+O\left(N^{2}\right) .
$$

Then for two critical particles $\gamma_{c . m}$. is also bounded, but the relative Lorentz factor at the collision event of a critical (1) particle and a usual (2) particle is

$$
m_{1} m_{2} \gamma_{c . m .}=\frac{X_{H}^{(2)}\left(X_{N}^{(1)}-Z_{N}^{(1)}\right)}{N}+O(1) \rightarrow \infty
$$

So, the BSW effects occurs whenever one usual and one critical particle collide near the horizon. Geodesic particles can be critical just due to the choice of initial conditions which fix $E$ and $L$, so one can always achieve $X_{H}=0$. The question is how resilient is the criticality attribute with respect to acceleration: whether a particle can remain critical under the action of finite forces, such as radiation reaction.

\section{E. Generalization: usual, critical and sub-critical particles}

In the absence of external forces acting on a particle, in the vicinity of a regular horizon, where all metric functions can be expanded into series by the radial coordinate $r$, the geodesic equation induces the same type of expansions for the parameters of a particle, such as $X$ and $E$. Therefore there are only two principally different types of particles: usual and critical ones. If we want to take into account forces acting on a particle, however, we have to allow for more general setting. In particular, we assume that acceleration components in the proper frame of a particle and $X$ can behave as $\xi^{q}$ and $\xi^{p}$ respectively, with some real $q$ and $p$, where

$$
\xi \equiv r-r_{H}
$$

Hereafter we consider this reasonably general while still relatively simple model.

If a particle reaches the horizon, $X$ must tend to zero more slowly than $N$, so that $Z^{2}$ remains positive. This is only possible for $p \leq 1$. On the other hand, it is reasonable to restrict our consideration to finite $E$ and $L$, and thus $X$, so $p \geq 0$. Then there are three 
possible particle types, distinguished by $p$ in

$$
X \sim \xi^{p}
$$

1. $p=0$ : usual particles.

$$
X_{u}=x_{H}+x_{1} \xi+\ldots, \quad Z_{u}=X+O\left(\xi^{2}\right) .
$$

2. $p=1$ : critical particles.

$$
X_{c r}=x_{1} \xi+x_{2} \xi^{2}+\ldots, \quad Z_{c r}=O(\xi) .
$$

3. $p \in(0,1)$ : the intermediate case, which will be called sub-critical particles hereafter:

$$
X_{s c}=\alpha \xi^{p}\left(1+x_{1} \xi+\ldots\right), \quad Z_{s c}=X_{s c}+O\left(\xi^{2-p}\right)
$$

Their proper time of reaching the horizon $\sim \int d \xi / Z_{s c}$ is finite.

As shown above (11), for collision of two particles the relative Lorentz factor is

$$
\gamma_{c . m .}=\frac{X_{1} X_{2}-Z_{1} Z_{2}}{m_{1} m_{2} N^{2}}+O(1)
$$

For collision of two usual or two critical particles near extremal horizon, for which $N^{2} \sim \xi^{2}$ (13), we have $\gamma_{c . m .}=O(1)$; for usual and critical $\gamma_{c . m .} \sim 1 / \xi$. Likewise for usual and sub-critical one obtains

$$
\gamma_{c . m .} \sim \xi^{-p} \rightarrow \infty
$$

for critical and sub-critical

$$
\gamma_{c . m .} \sim \xi^{p-1} \rightarrow \infty
$$

So, the corresponding particles behave as critical in collisions with usual ones and as usual in collisions with critical ones. 
This result can be derived in the general setting. First of all, let there be a particle with

$$
\begin{aligned}
& X=\alpha \xi^{p}(1+O(\xi)), \quad p \in[0,1] \\
& \frac{L^{2}}{g_{\phi}}+m^{2}=\beta^{2}+O(\xi), \quad \alpha, \beta \sim 1 .
\end{aligned}
$$

Then

$$
Z-X=-C \xi^{2-p}(1+O(\xi))
$$

where

$$
C= \begin{cases}\beta^{2} / 2 \alpha & \text { for } \quad p<1 \\ \alpha-\sqrt{\alpha^{2}-\beta^{2}} & \text { for } \quad p=1\end{cases}
$$

As due to forward in time condition $\alpha>0$ and $\beta^{2}$ is also positive, $C$ is strictly positive as well.

Now suppose we have two such particles, with $p_{1}$ and $p_{2}$, colliding near the horizon. Then using (35) and (40), the relative Lorentz factor is reduced to

$$
\begin{aligned}
m_{1} m_{2} \gamma_{c . m .} & =O(1)+(1+O(\xi))\left[C_{1} \alpha_{2} \xi^{p_{2}-p_{1}}+C_{2} \alpha_{1} \xi^{p_{1}-p_{2}}\right] \\
& \sim \xi^{-\left|p_{1}-p_{2}\right|}\left[1+O(\xi)+O\left(\xi^{2\left|p_{1}-p_{2}\right|}\right)\right]
\end{aligned}
$$

and thus

$$
\gamma_{c . m .} \sim \xi^{-\left|p_{1}-p_{2}\right|}
$$

Here, gamma becomes finite only if $p_{1}=p_{2}$. We see that consideration of sub-critical particles is convenient, as it allows to describe usual and critical particles in a more coherent and unified way, while at the same time providing greater generality, necessary when dealing with non-geodesic motions.

\section{DYNAMICS}

\section{A. OZAMO and FZAMO frames}

There are two main qualitatively different frames of reference in the vicinity of a black hole horizon. The tetrad vectors and tetrad components of different quantities will be denoted 
by superscripts in parenthesis, while low case "o" or "f" in the subscript will denote which frame is used, i.e. $a_{o}^{(t)}$ is the $t$-component of acceleration in the OZAMO frame (see below).

a. OZAMO. The first kind of frame is attached to an observer who is orbiting the black hole with constant $r$, having constant energy and zero angular momentum. We will call it OZAMO for orbital zero angular momentum observer ${ }^{4}$. It is the analogue of the static observer in a static spacetime, and it becomes lightlike in the horizon limit [14].

The tetrad 1-forms of the OZAMO frame, denoted by small "o" subscripts, read

$$
\begin{aligned}
& e_{o}^{(t)}=-N d t \\
& e_{o}^{(\phi)}=g_{\phi}^{1 / 2}(d \phi-\omega d t) ; \\
& e_{o}^{(r)}=A^{-1 / 2} d r \\
& e_{o}^{(z)}=g_{z}^{1 / 2} d z .
\end{aligned}
$$

If another particle's four-velocity is $u^{\mu}$, then its Lorentz factor in this frame is

$$
\gamma=-u^{\mu}\left(e_{o}^{(t)}\right)_{\mu}=\frac{X}{m N}
$$

Thus for a particle with $X \sim \xi^{p}$

$$
\gamma \sim \xi^{p-1}
$$

for a usual particle it diverges in the horizon limit, while for a critical one it stays finite.

A particle's acceleration is

$$
a^{\mu} \equiv u^{\nu} \nabla_{\nu} u^{\mu}
$$

Its tetrad components in the OZAMO frame

$$
a_{o}^{(i)}=a^{\mu}\left(e_{o}^{(i)}\right)_{\mu}, \quad i=t, \phi, r, z,
$$

\footnotetext{
4 This observer is usually called just ZAMO in textbooks, but we need to be more specific.
} 
are equal to

$$
\begin{aligned}
& a_{o}^{(t)}=N a^{t} ; \\
& a_{o}^{(\phi)}=\sqrt{g_{\phi}}\left(a^{\phi}-\omega a^{t}\right) ; \\
& a_{o}^{(r)}=\frac{1}{\sqrt{A}} a^{r} ; \\
& a_{o}^{(z)}=\sqrt{g_{z}} a^{z}
\end{aligned}
$$

the acceleration scalar then can be presented as

$$
a^{2} \equiv a^{\mu} a_{\mu}=-\left(a_{o}^{(t)}\right)^{2}+\left(a_{o}^{(\phi)}\right)^{2}+\left(a_{o}^{(r)}\right)^{2}+\left(a_{o}^{(z)}\right)^{2} .
$$

The OZAMO orbits the horizon at constant $r$ and does not cross it, therefore it is not classified as either usual or critical particle, which does cross or approach the horizon in infinite proper time respectively. However, it is useful to note, that, as its Lorentz factor is finite with respect to a critical particle, and vice versa, in the discussion that follows, OZAMO and critical particles behave similarly.

It is well-known, that an OZAMO frame breaks down at the horizon, thus strictly speaking at the horizon it is not a valid frame and OZAMO is not an observer in the traditional sense. Hereafter, what we refer to as the values of some quantities measured in the OZAMO frame in the horizon limit are the limits of the corresponding quantities measured in successive different OZAMO frames, with different $r_{Z A M O}$, when $r_{Z A M O} \rightarrow r_{H}$.

b. FZAMO. The other important frame of reference is realized by one of the usual particles crossing the horizon. For simplicity, it is convenient to take for such an observer $L=0$ similarly to OZAMO and, additionally, $E=m$. Thus we will call the corresponding observer FZAMO for falling zero angular momentum observer. Its frame $\left\{e_{f}^{(i)}\right\}$, with $i=$ $t, \phi, r, z$, is constructed by making a local Lorentz transformation from the OZAMO in the 
direction towards the horizon ${ }^{5}$ :

$$
\left(\begin{array}{c}
\left(e_{f}^{(t)}\right)_{\mu} \\
\left(e_{f}^{(r)}\right)_{\mu}
\end{array}\right)=\gamma_{f}\left(\begin{array}{cc}
1 & v_{f} \\
v_{f} & 1
\end{array}\right)\left(\begin{array}{c}
\left(e_{o}^{(t)}\right)_{\mu} \\
\left(e_{o}^{(r)}\right)_{\mu}
\end{array}\right)
$$

The FZAMO's Lorentz factor in the OZAMO frame is $\gamma_{f}=-\left(u^{\mu}\right)\left(e_{o}^{(t)}\right)_{\mu}$, where $u^{\mu}$ is given by (2) with $L=0$ and $E=m$, and $v_{f}=\sqrt{1-\gamma_{f}^{-2}}$.

Then,

$$
\gamma_{f}=\frac{1}{N}, \quad v_{f}=\sqrt{1-N^{2}}
$$

The corresponding tetrad components of acceleration

$$
a_{f}^{(i)}=a^{\mu}\left(e_{f}^{(i)}\right)_{\mu}, \quad i=t, \phi, r, z
$$

are related to $a_{o}^{(i)}$ by the respective Lorentz transformation which becomes singular on the horizon, where $N \rightarrow 0, \gamma_{f} \rightarrow \infty$.

c. Proper frame. For non-critical particles with $p<1$ the Lorentz factor relative to the OZAMO frame diverges as $\gamma \sim \xi^{p-1}(37)$. Thus the correct reference frame for it will have the same behaviour of Lorentz factor. We will construct it, analogously to FZAMO, by making the corresponding boost in the radial direction, and call it for simplicity the proper frame for a particle, although it may not be exactly proper. What is important is that, in contrast to the OZAMO, the particle's velocity in it stays finite (does not tend to $c$ ).

Thus, given a particle's Lorentz factor in the OZAMO frame, $\gamma$ (without subscripts), the tetrad components of acceleration in the proper frame are

$$
\left(\begin{array}{c}
a_{p r}^{(t)} \\
a_{p r}^{(r)}
\end{array}\right)=\gamma\left(\begin{array}{ll}
1 & v \\
v & 1
\end{array}\right)\left(\begin{array}{c}
a_{o}^{(t)} \\
a_{o}^{(r)}
\end{array}\right)
$$

For a usual or critical particle this reduces to the already considered OZAMO and FZAMO frames respectively, while for sub-critical particles the proper frame does not coincide with either one of those.

\footnotetext{
${ }^{5}$ Note that this is the transformation for one-forms; vectors are transformed by the inverse matrix, which differs by the sign of $v_{f}$.
} 


\section{B. Acceleration in different frames}

When describing particles' motion near the horizon, we must restrict ourselves to particles with finite acceleration. This necessarily means that the acceleration scalar $a^{2}$ should be finite. It would seem that it is natural to demand that tetrad components of acceleration are finite as well. However, as shown above, when we describe a particles' motion near the horizon, we have different frames of reference, which are related to each other by singular Lorentz transformations. This means that finite tetrad components of acceleration in one of the frames may correspond to diverging tetrad components in the other or vice versa.

The frame in which tetrad components of a particle's acceleration should be finite is the instantly comoving frame, or equivalently, any frame which moves with finite Lorentz factor with respect to that. For example, recall the reasonably realistic problem of a charged particle in a uniform electric field in Special Relativity. The tetrad components of acceleration in the laboratory frame (with Minkowski metric) diverge proportionally to the Lorentz factor, while those in the instantly comoving frame (with the Rindler metric and the horizon) are constant (see, e.g. p. 403 of [15]).

For a critical particle then acceleration is adequately and most easily measured in the OZAMO frame. For a usual particle we would have to attach the tetrad also to one of the usual particles, for example to FZAMO. Due to normalization of four-velocity $u^{\mu} u_{\mu}=-1$, which implies $a^{\mu} u_{\mu}=0$, in each case it is sufficient to show that three of the four tetrad components are finite.

\section{Energy and angular momentum}

If $\xi^{\mu}$ is a Killing vector field, then

$$
\frac{d}{d \tau}\left(\xi^{\mu} u_{\mu}\right)=\xi^{\mu} a_{\mu}
$$


In a stationary axisymmetric metric we have two Killing vectors $\xi_{t}^{\mu}=\delta_{t}^{\mu}$ and $\xi_{\phi}^{\mu}=\delta_{\phi}^{\mu}$, which give

$$
\begin{aligned}
& \frac{1}{m} \frac{d E}{d \tau}=\left(N^{2}-\omega^{2} g_{\phi}\right) a^{t}+\omega g_{\phi} a^{\phi} \\
& \frac{1}{m} \frac{d L}{d \tau}=-\omega g_{\phi} a^{t}+g_{\phi} a^{\phi}
\end{aligned}
$$

or through the tetrad components in the OZAMO frame $(52,53)$

$$
\begin{aligned}
& \frac{1}{m} \frac{d E}{d \tau}=N a_{o}^{(t)}+\omega \sqrt{g_{\phi}} a_{o}^{(\phi)} \\
& \frac{1}{m} \frac{d L}{d \tau}=\sqrt{g_{\phi}} a_{o}^{(\phi)} .
\end{aligned}
$$

It is clear, that if the proper time of crossing the horizon is finite, as is the case for the usual particles, then the finiteness of $N a_{o}^{(t)}$ and $a_{o}^{(\phi)}$ implies that $E$ and $L$ are also bounded. However, this does not seem to be necessarily so for critical particles, for which the proper time of reaching the horizon diverges.

\section{Dynamic restrictions on a particle's velocity}

Let us enumerate and classify all the possible variants of particle's type of asymptotic motion in the vicinity of the horizon, now in more detail than in the section on kinematics, so as to focus below only on those that are not explicitly non-physical.

First of all, diverging $L$, as seen from (65), would correspond to continuous acceleration in the $\phi$ direction, which would cost formally infinitely large amounts of fuel per a unit mass particle. If one has the resources to make such experiments, he would not need the BSW effect in order to observe (formally) infinite energy in the center of mass frame. So this variant is of not much interest.

Secondly, one could imagine divergent $u^{z}$. Such a particle would have velocity tending to $c$ and directed along the $z$ axis (or at finite angle with respect to it) both in the OZAMO and FZAMO frames. This would mean that the particle is "accelerated" (in the sense that its velocity increases) not only in radial direction, but also along the horizon surface. This would be very strange behaviour, and in the Kerr metric such particles are naturally absent [16]. We will not consider this variant here. 
Given these two natural assumptions, from the normalizing condition

$$
Z^{2}=X^{2}-N^{2} \tilde{\beta}^{2}
$$

where $\tilde{\beta}$ in the horizon limit tends to a positive real number, finite and separated from zero. Consequently, for a particle reaching the horizon, where $Z^{2}$ must remain positive, and at the same time finite $X$ and $E$ (see discussion after Eq. (30)), we have

$$
X \sim \xi^{p}, \quad \text { with } \quad p \in[0,1]
$$

which corresponds to usual, sub-critical and critical particles as discussed above.

\section{E. Usual particles}

For a usual particle $X_{H} \neq 0$ by definition. As discussed above, the tetrad components of its acceleration in the FZAMO frame $a_{f}^{(i)}$ must be finite. Then the components in the OZAMO frame $a_{o}^{(i)}$, related to them via the singular Lorentz transform (58), with $\gamma_{f}=1 / N$, can diverge as $1 / N$. Writing out explicitly the asymptotics for the $t$ and $r$ components in both frames, we get

$$
\begin{aligned}
& a_{f}^{(t)}=\left(a_{f}^{(t)}\right)_{0}+\left(a_{f}^{(t)}\right)_{1} N+O\left(N^{2}\right) ; \\
& a_{f}^{(r)}=\left(a_{f}^{(r)}\right)_{0}+\left(a_{f}^{(r)}\right)_{1} N+O\left(N^{2}\right) ; \\
& a_{o}^{(t)}=+\frac{\left(a_{f}^{(t)}\right)_{0}-\left(a_{f}^{(r)}\right)_{0}}{N}+\left[\left(a_{f}^{(t)}\right)_{1}-\left(a_{f}^{(r)}\right)_{1}\right]+O(N) ; \\
& a_{o}^{(r)}=-\frac{\left(a_{f}^{(t)}\right)_{0}-\left(a_{f}^{(r)}\right)_{0}}{N}-\left[\left(a_{f}^{(t)}\right)_{1}-\left(a_{f}^{(r)}\right)_{1}\right]+O(N),
\end{aligned}
$$

The $\phi$ and $z$ components are the same in the two frames and must be bounded:

$$
\begin{aligned}
& a_{f}^{(\phi)}=a_{o}^{(\phi)}=O(1) ; \\
& a_{f}^{(z)}=a_{o}^{(z)}=O(1) .
\end{aligned}
$$

Then, we see that if $a_{f}^{(i)}=O(1)$, the right hand side of (64) is finite. The left hand side is also finite, as for a usual particle, given $u^{r} \sim 1$ (18), $d r \sim d \tau \sim d N$. 
The explicit expressions for $a_{o}^{(r)}$ and $a_{o}^{(z)}$ are

$$
\begin{aligned}
a_{o}^{(r)}= & \frac{1}{\sqrt{A}}\left\{\left(u^{r} \partial_{r}+u^{z} \partial_{z}\right) u^{r}-\frac{A^{\prime}}{2 A}\left(u^{r}\right)^{2}-\frac{A}{2} \partial_{r} g_{z}\left(u^{z}\right)^{2}+\right. \\
& \left.-\frac{A}{2}\left[X^{2} \partial_{r} N^{-2}-L^{2} \partial_{r} g_{\phi}^{-1}-2 \frac{X L}{N^{2}} \partial_{r} \omega\right]\right\} ; \\
a_{o}^{z}= & \sqrt{g_{z}}\left\{\left(u^{r} \partial_{r}+u^{z} \partial_{z}\right) u^{z}+\frac{\partial_{z} g_{z}}{2 g_{z}}\left(u^{z}\right)^{2}+\frac{\partial_{r} g_{z}}{g_{z}} u^{r} u^{z}+\right. \\
& \left.-\frac{1}{2 g_{z}}\left[X^{2} \partial_{z} N^{-2}-L^{2} \partial_{z} g_{\phi}^{-1}-2 \frac{X L}{N^{2}} \partial_{z} \omega\right]\right\} .
\end{aligned}
$$

The conditions $a_{f}^{(i)}=O(1)$ can be reformulated in the form of restrictions on the coefficients $\alpha_{k}$ and $\beta_{k}$ in the expansions

$$
\begin{aligned}
& u^{r}=\alpha_{0}(z)+\alpha_{1}(z)\left(r-r_{H}\right)+O\left(\left(r-r_{H}\right)^{2}\right), \\
& u^{z}=\beta_{0}(z)+\beta_{1}(z)\left(r-r_{H}\right)+O\left(\left(r-r_{H}\right)^{2}\right) .
\end{aligned}
$$

\section{F. Critical particles}

Such particles approach the horizon but, in contrast to usual ones, the process takes infinite proper time. On the other hand, as seen from (19) and (49), their Lorentz factor in the OZAMO frame $\gamma$ is finite, and the velocity is $v<1$, so the tetrad components of acceleration in the OZAMO frame must be finite. As mentioned above, we consider only motion with $E$ and $L$ bounded in the horizon limit $\tau \rightarrow \infty$. This means that $a_{o}^{(\phi)}$ should be not only bounded, but integrable (65): $\int d \tau a_{o}^{(\phi)}<\infty$. If we assume that $a_{o}^{(\phi)}$ is expandable in power series by $r$ with integer powers, this means

$$
a_{o}^{(\phi)}=O(N)
$$

With this condition satisfied, and Eq. (70) taken into account, the boundedness of $E$ from (64) does not give any more restrictions on $a_{o}^{(t)}$. Then, using that $E, L$, and $u^{z}$ are bounded (15), while $u^{r}$ and $X$ are $O(N)$, and $\omega_{H}=$ const (which follows from regularity [13]), it is easy to see that all the terms in (74) and (75) are automatically finite, so

$$
a_{o}^{(r)}, a_{o}^{(z)}=O(1)
$$


Thus all components of acceleration of a critical particle in the OZAMO frame, and therefore in the instantly comoving proper frame, are finite unconditionally. This is in contrast to usual particles, for which the conditions $a_{f}^{(i)}=O(1)$ impose some additional constraints on $\alpha_{k}$ and $\beta_{k}$ in (76).

In the FZAMO frame, and the frame of any usual particle, the picture looks different, as the relative Lorentz factor of a usual and critical particle diverges as $1 / N$. Using the Lorentz transformation (57) between the OZAMO and FZAMO frames, with $\gamma_{f} \sim 1 / N$, we see that $a_{f}^{(t)}$ and $a_{f}^{(r)}$ can diverge as $1 / N$. Using (57), the asymptotics of these components of acceleration in the two frames can be brought to the form

$$
\begin{aligned}
& a_{o}^{(t)}=\left(a_{o}^{(t)}\right)_{0}+\left(a_{o}^{(t)}\right)_{1} N+O\left(N^{2}\right) ; \\
& a_{o}^{(r)}=\left(a_{o}^{(r)}\right)_{0}+\left(a_{o}^{(r)}\right)_{1} N+O\left(N^{2}\right) ; \\
& a_{f}^{(t)}=\frac{\left(a_{o}^{(t)}\right)_{0}+\left(a_{o}^{(r)}\right)_{0}}{N}+\left[\left(a_{o}^{(t)}\right)_{1}+\left(a_{o}^{(r)}\right)_{1}\right]+O(N) ; \\
& a_{f}^{(r)}=\frac{\left(a_{o}^{(t)}\right)_{0}+\left(a_{o}^{(r)}\right)_{0}}{N}+\left[\left(a_{o}^{(t)}\right)_{1}+\left(a_{o}^{(r)}\right)_{1}\right]+O(N) .
\end{aligned}
$$

The $\phi$ and $z$ components in the two frames are the same and therefore, as shown above, satisfy

$$
\begin{aligned}
& a_{o}^{(\phi)}=a_{f}^{(\phi)}=O(N) ; \\
& a_{o}^{(z)}=a_{f}^{(z)}=O(1) .
\end{aligned}
$$

Thus we have two mutually complimentary cases. In the OZAMO frame $r$ and $t$ components of acceleration diverge for usual particles and stay finite for the critical ones. In the FZAMO frame, the situation is opposite: $r$ and $t$ components of acceleration are finite for usual particles and diverge for the critical ones. The $\phi$ and $z$ components are the same in the two frames and are finite. For critical particles, additionally $a_{o}^{(\phi)}=O(N)$ near the horizon for energy and angular momentum to remain bounded. 


\section{EXAMPLE: THE REISSNER-NORDSTRÖM METRIC}

The approach and results of the present paper are also valid in the case of the electromagnetic interaction with minimal changes: in eq. (4) one should make the replacement $X \rightarrow X-q \varphi$, where $\varphi$ is the electrostatic potential, and $q$ is the particle's charge. In

order to demonstrate this, it is instructive to consider as an example the extremal ReissnerNordström metric. In this case the metric functions are

$$
N=\sqrt{A}=1-\frac{r}{r_{H}}, \quad \omega=0, \quad g_{\phi}=r^{2},
$$

and the electromagnetic field potential is

$$
A_{\mu}=-\varphi \delta_{\mu}^{t}, \quad \varphi=\frac{Q}{r}
$$

where

$$
Q=r_{H}
$$

is the extremal black hole's charge, so that the only nonvanishing components of the electromagnetic field tensor are

$$
F_{r t}=-F_{t r}=\frac{Q}{r^{2}}
$$

For a particle of charge $q$ moving radially towards the horizon the four-momentum can be parametrized as

$$
p_{\mu}=m u_{\mu}=-\left(X, 0, Z / N^{2}, 0\right),
$$

then the normalization condition implies

$$
Z=\sqrt{X^{2}-m^{2} N^{2}} .
$$

The equation of motion

$$
m a^{\mu}=q F^{\mu \nu} u_{\nu}
$$

has the integral of motion

$$
E=X+q \varphi=\text { const }
$$


For usual particles, with $X_{H} \neq 0$,

$$
p^{r}=-X_{H}-\frac{q}{r_{H}}\left(r-r_{H}\right)+O\left(\left(r-r_{H}\right)^{2}\right),
$$

in agreement with (76).

For a critical particle

$$
E=q, \quad X=q N, \quad Z=N \sqrt{q^{2}-m^{2}} .
$$

Then, integrating the equation for radial motion

$$
\frac{d r}{d \tau}=-\frac{Z}{m}=-N(r) \sqrt{q^{2} / m^{2}-1}
$$

it is easy to obtain that in the horizon limit the same asymptotic as in eq. (22) holds, with the characteristic time

$$
\tau_{0}=r_{H}\left(\frac{q^{2}}{m^{2}}-1\right)^{-1 / 2}
$$

Now, we will consider the acceleration measured by the two types of observers.

\section{A. Static observers}

The tetrad (45)-(48) in this case turns into the tetrad of a static observer. Then, using (45) and (52), we obtain

$$
\begin{gathered}
m a_{o}^{(t)}=-\frac{q Q}{r^{2}} \frac{Z}{m N} \\
m a_{o}^{(r)}=+\frac{q Q}{r^{2}} \frac{X}{m N} \\
m^{2} a^{2}=\left(\frac{q Q}{r^{2}}\right)^{2} .
\end{gathered}
$$

For the critical particle (93) both components of acceleration

$$
\begin{aligned}
m a_{o}^{(t)} & =-\frac{q Q}{r^{2}} \sqrt{q^{2} / m^{2}-1}, \\
m a_{o}^{(r)} & =\frac{q Q}{r^{2}} \frac{E}{m}
\end{aligned}
$$


are finite on the horizon, and can be expanded into a series by $\left(r-r_{H}\right)$ or $N$.

However, for a usual particle, with $X_{H} \neq 0$, near the horizon

$$
X=X_{H}+O(N), \quad Z=X+O\left(N^{2}\right),
$$

SO

$$
a_{o}^{(r)} \approx-a_{o}^{(t)}=\frac{a_{-1}}{N}+O(1)
$$

where

$$
a_{-1}=\frac{q}{Q} \frac{X_{H}}{m^{2}}
$$

Thus both components diverge near the horizon, in accordance with $(71,70)$, while satisfying

$$
a_{o}^{(r)}+a_{o}^{(t)}=O(N)
$$

\section{B. Falling observers}

The falling frame $e_{f}^{(i)}$ is attached to a particle falling into the black hole according to (57)-(58), with the Lorentz factor $\gamma_{f}=1 / N$ and velocity $v_{f}=1-O\left(N^{2}\right)$ in the static frame.

In this frame the tetrad components of acceleration are equal to

$$
\begin{aligned}
& m a_{f}^{(t)}=-\gamma_{F} \frac{q Q}{r^{2}} \frac{Z-v_{F} X}{m N} \\
& m a_{f}^{(r)}=+\gamma_{F} \frac{q Q}{r^{2}} \frac{X-v_{F} Z}{m N}
\end{aligned}
$$

For usual particles, in the horizon limit $N \rightarrow 0, X_{H} \neq 0$. Then (58) and (89) imply that $Z-X=O\left(N^{2}\right)$, so $a_{f}^{(t)}$ and $a_{f}^{(r)}$ are finite.

If the particle under consideration is critical, then $Z \sim X \sim N$, and both components of 
acceleration diverge:

$$
\begin{aligned}
a_{f}^{(t)}= & \frac{\tilde{a}_{-1}}{N}+O(N), \\
a_{f}^{(r)}= & \frac{\tilde{a}_{-1}}{N}+O(N), \\
& \tilde{a}_{-1}=r_{H}^{-1} \frac{q}{m}\left[\frac{q}{m}-\sqrt{\frac{q^{2}}{m^{2}}-1}\right] .
\end{aligned}
$$

Thus we see that, indeed, all the general properties (68), (69), (70), (71), described in the preceding section, are explicitly verified in this exactly solvable case.

\section{BSW EFFECT UNDER FINITE FORCES: EQUATORIAL MOTION}

\section{A. Motion in equatorial plane}

Let $m=1$, and let us consider motion in the equatorial plane so that $u^{z}=0$ and $a_{o}^{(z)}=0$. Then for arbitrary motion we have 1) the normalization condition for velocity

$$
u^{r}=-\frac{\sqrt{A}}{N} Z, \quad Z=\sqrt{X^{2}-N^{2}\left[\frac{L^{2}}{g_{\phi}}+1\right]},
$$

and 2) orthogonality condition for acceleration, which can be written in terms of (52-55) as

$$
\begin{aligned}
0=u_{\mu} a^{\mu} & =+u_{t} a^{t}+u_{\phi} a^{\phi}+u_{r} a^{r} \\
& =-E a^{t}+L a^{\phi}+A^{-1} u^{r} a^{r} \\
& =-\frac{X}{N} a_{o}^{(t)}+\frac{L}{\sqrt{g_{\phi}}} a_{o}^{(\phi)}+\frac{u^{r}}{\sqrt{A}} a_{o}^{(r)} .
\end{aligned}
$$

Generically, at least two of the three components of $a^{(i)}$ have to be non-zero if there is acceleration. Also for simplicity we will assume ${ }^{6}$ that $A=N^{2}$, so that $u^{r}=-Z$ and

\footnotetext{
${ }^{6}$ This assumption is purely technical. In general, one should write $A=N^{2} B$, where $B$ is some bounded function which does not vanish at the horizon. Its form does not affect the results qualitatively but leads to more cumbersome expressions. Thus we put for simplicity $B=1$, which also fixes the time scale.
} 
orthogonality condition takes form

$$
\frac{X}{N} a_{o}^{(t)}-\frac{L}{\sqrt{g_{\phi}}} a_{o}^{(\phi)}+\frac{Z}{N} a_{o}^{(r)}=0 .
$$

Of the four components of the equation of motion

$$
\left(u^{\mu} \nabla_{\mu}\right) u^{\nu}=a^{\nu}
$$

one is trivial ${ }^{7} a^{z}=0$, and the other three are related through the orthogonality condition, so it is always sufficient to consider only two components, for example (64) and (65), which can be written in terms of $X$ and $L$ as

$$
\begin{aligned}
& \frac{d X}{d \tau}=N a_{o}^{(t)}-L \frac{d \omega}{d \tau} \\
& \frac{d L}{d \tau}=\sqrt{g_{\phi}} a_{o}^{(\phi)}
\end{aligned}
$$

As $d r / d \tau=u^{r}=-Z$, in terms of $X$ and derivatives by $\xi \equiv\left(r-r_{H}\right)$, which are denoted by primes, this can be written as

$$
\begin{aligned}
& X^{\prime}+L \omega^{\prime}=-\frac{N}{Z} a_{o}^{(t)} ; \\
& L^{\prime}=-\frac{\sqrt{g_{\phi}}}{Z} a_{o}^{(\phi)} .
\end{aligned}
$$

It can be checked that, indeed, in case $u^{z}=0$, equations (114) and (115) together with (112) give (74).

$\overline{7}$ In the equatorial plane derivatives of metric functions by $z$ in (75) must vanish due to symmetry. 


\section{B. Acceleration in proper frame}

Expressing acceleration components in the OZAMO frame through the particle's parameters $E$ and $L$ from $(116,117)$ and the orthogonality condition (112), one obtains

$$
\begin{aligned}
a_{o}^{(\phi)} & =-\frac{Z}{\sqrt{g_{\phi}}} L^{\prime} ; \\
a_{o}^{(t)} & =-\frac{Z}{N}\left(X^{\prime}+L \omega^{\prime}\right) ; \\
a_{o}^{(r)} & =-\frac{X}{Z} a_{o}^{(t)}-N \frac{L L^{\prime}}{g_{\phi}} .
\end{aligned}
$$

For critical particles the OZAMO frame is the proper frame. For other types of particles the $r$ and $t$ components of acceleration in the proper frame are given by (60) with Lorentz factor $(49)$

$$
\gamma=\frac{X}{N}
$$

while $a_{p r}^{(\phi)}=a_{o}^{(\phi)}$ for any type. Using (118-120), this gives

$$
\left(\begin{array}{c}
a_{p r}^{(t)} \\
a_{p r}^{(r)}
\end{array}\right)=\frac{X}{N}\left\{\frac{a_{o}^{(t)}}{Z}\left(\begin{array}{c}
Z-v X \\
Z v-X
\end{array}\right)-N\left(\begin{array}{l}
v \\
1
\end{array}\right) \frac{L L^{\prime}}{g_{\phi}}\right\}
$$

Suppose we have a particle with

$$
\begin{aligned}
& X=\alpha \xi^{p}(1+O(\xi)), \quad \gamma=\frac{X}{\xi} \\
& v=\sqrt{1-\gamma^{-2}}=1-\frac{1}{2 \alpha} \xi^{2(1-p)}(1+O(\xi)),
\end{aligned}
$$

where $p<1$. Using (40), we get

$$
\begin{aligned}
& Z-X v=\left(\frac{1}{2}-C\right) \xi^{2-p}(1+O(\xi)), \\
& X-Z v=\left(\frac{1}{2}+C\right) \xi^{2-p}(1+O(\xi)) .
\end{aligned}
$$

\section{Usual particles}

For a usual particle $p=0$, so assuming $L^{\prime}$ is bounded, $a_{o}^{(\phi, r, t)}=O(1)$, while $\gamma \sim 1 / \xi$, and one can easily check term by term that acceleration in the proper frame (122) is always 
bounded: as expected, for usual particles there are no additional requirements.

\section{Sub-critical particles}

For a sub-critical particle $p \in(0,1)$. As $X \sim Z$, the derivative $X^{\prime} \sim \xi^{p-1}$ in (119) diverges, while $L \omega^{\prime}=O(1)$, so

$$
\frac{a_{o}^{(t)}}{Z} \sim \xi^{p-2} .
$$

Then taking into account (125-126), the first term in the braces of $(122)$ is $O(1)$, and different in the two rows, thus separated from zero.

The second term could only compensate the first one (in one of the two rows), if $L^{\prime} \sim \xi^{-1}$, which would imply divergent $L \sim \ln \xi$. Therefore the quantity in the braces is finite and separated from zero, so the proper acceleration diverges as (121)

$$
\gamma=\frac{X}{N} \sim \xi^{p-1} \rightarrow \infty
$$

This means that there are no sub-critical particles with finite acceleration for motion in the equatorial plane.

\section{Critical particles}

The only remaining case to be considered is critical particles. Although (122) for them is unnecessary, one restores the acceleration in the OZAMO frame from it by setting $\gamma=1$ and $v=0$. We see that ${ }^{8}$

$$
\begin{aligned}
a_{o}^{(\phi)} & \sim \xi L^{\prime} ; \\
a_{o}^{(r, t)} & \sim\left(X^{\prime}+L \omega^{\prime}\right),
\end{aligned}
$$

so in order for such trajectory to be realized we need the azimuthal force to tend to zero fast enough:

$$
a_{o}^{(\phi)}=O(\xi)
$$

\footnotetext{
${ }^{8}$ Remember that components $a_{o}^{(i)}$ are related through the orthogonality condition (112); if two of them are finite, then the third is bounded as well.
} 
There is no restriction on the radial component: it can be of the order of unity, as it will still be possible to fine-tune a critical particle by the appropriate choice of initial condition (this will be shown in more detail in the next Section). Thus the radial component does not affect or hinder the existence of critical trajectories and consequently the BSW effect. This is in agreement with the already established fact that the radial force itself is the reason for the BSW effect near charged nonrotating black holes [19].

\section{Example: azimuthal dissipative force}

Let us consider the particular case when the radial force, which does not hinder critical particles anyway, is absent:

$$
a_{o}^{(r)}=0, \quad a_{o}^{(t)}, a_{o}^{(\phi)} \neq 0 .
$$

Using orthogonality (112),

$$
a_{o}^{(t)}=\frac{N}{X} \frac{L}{\sqrt{g_{\phi}}} a_{o}^{(\phi)},
$$

so in terms of derivatives with respect to $\xi$ Eqs. (116) and (117) can be rewritten as

$$
\begin{aligned}
& g_{\phi} X\left(X^{\prime}+L \omega^{\prime}\right)=N^{2} L L^{\prime} ; \\
& a_{o}^{(t)}=-N \frac{Z}{X} \frac{L L^{\prime}}{g_{\phi}} \\
& a_{o}^{(\phi)}=-Z \frac{L^{\prime}}{\sqrt{g_{\phi}}} .
\end{aligned}
$$

\section{Tuning a critical particle}

In this section we show in more detail how one would tune the particle to be critical $X \sim \xi$. 
Assuming expansions

$$
\begin{aligned}
N^{2} & =\nu_{2} \xi^{2}+\nu_{3} \xi^{3}+\ldots, \\
\omega & =\omega_{H}-\omega_{1} \xi+\omega_{2} \xi^{2}+\ldots, \\
g_{\phi} & =g_{H}+g_{1} \xi+g_{2} \xi^{2}+\ldots, \\
X & =x_{1} \xi+x_{2} \xi^{2}+\ldots \\
L & =l_{H}+l_{1} \xi+l_{2} \xi^{2}+\ldots,
\end{aligned}
$$

from (134) we obtain in consecutive orders

$$
\begin{aligned}
l_{H} & =\frac{x_{1}}{\omega_{1}} \\
l_{1} & =2 \frac{x_{1} \omega_{2}+x_{2} \omega_{1}}{\omega_{1}^{2}+\nu_{2} / g_{H}} ; \\
l_{2} & =l_{2}\left(x_{1}, x_{2}, x_{3}\right), \quad \ldots
\end{aligned}
$$

Then

$$
\frac{Z^{2}}{\xi^{2}} \approx x_{1}^{2}\left[1-\frac{\nu_{2}}{g_{H}} \omega_{1}^{-2}\right]-\nu_{2}
$$

There is a critical particle for

$$
\left|x_{1}\right|>x_{1 \min }
$$

and there is a solution

$$
x_{1 \min }=\frac{\nu_{2}}{1-\frac{\nu_{2}}{g_{H}} \omega_{1}^{-2}}
$$

as long as

$$
\omega_{1}^{2}>\frac{\nu_{2}}{g_{H}}
$$

From (135) we get

$$
a_{o}^{(t)}=-\frac{Z}{N}\left(X^{\prime}+L \omega^{\prime}\right)
$$

and after substitution of expansions for $X, L$ and $\omega$,

$$
a_{o}^{(t)} \approx-\frac{2 Z}{\omega_{1} \sqrt{\nu_{2}}} \frac{x_{1} \omega_{2}+x_{2} \omega_{1}}{1+g_{H} \omega_{1}^{2} / \nu_{2}} \sim Z \sim \xi
$$

Further terms are obtained straightforwardly but they are quite cumbersome. 
So, if acceleration is expanded in a series by $\xi$

$$
a_{o}^{(t)}=a_{1} \xi+a_{2} \xi^{2}+\ldots
$$

in the first order we obtain $a_{1}\left(x_{1}, x_{2}\right)$. As long as $a=O(\xi)$, and the metric coefficients satisfy (148), we can take arbitrary $x_{1}$ such that $\left|x_{1}\right|>x_{1 \text { min }}$ (or equivalently $l_{H}=x_{1} / \omega_{1}$ ). Then for the given $a_{1}$ in the first order we obtain $x_{2}\left(a_{1}\right)$, in the next order $x_{3}\left(a_{1}, a_{2}\right)$ and so on. The set of critical trajectories ${ }^{9}$ is parametrized by one free parameter $x_{1}\left(\right.$ or $\left.l_{H}\right)$.

\section{Other realizations of critical trajectories}

Suppose now the azimuthal force tends to zero as $\xi^{s}$ with some integer $s>1$. Then from (136) we see that $L^{\prime} \sim \xi^{s-1}$, and therefore expansion (15) for $L$ near the horizon takes the form

$$
L=L_{H}+L_{s} \xi^{s}(1+o(1))
$$

It is perfectly consistent with the particle being critical, so that $X \sim \xi$ : (134) can be satisfied for any integer $s>1$ and solved for $X(L)$ (or, equivalently, $E(L)$ ) in each consecutive order by $\xi$. Let us consider, for example, the case $s=2$. Assuming

$$
X=x_{1} \xi+x_{2} \xi^{2}+x_{3} \xi^{3}+O\left(\xi^{4}\right)
$$

from (134) in consecutive orders one obtains

$$
\begin{aligned}
& x_{1}=L_{H} \omega_{1} ; \\
& x_{2}=-2 \omega_{2} L_{H} ; \\
& x_{3}=-\omega_{3} L_{H}+\frac{\omega_{1}}{3} L_{2}+\frac{2}{3 g_{\phi H}} L_{H} L_{2} ;
\end{aligned}
$$

\footnotetext{
${ }^{9}$ For large enough $\left|x_{1}\right|$ the turning point, given by $Z=0$, will be at finite values of $\left(r-r_{H}\right)$ from the horizon. Thus it will be at the coordinate distance that does not have to be small in order to gain arbitrarily large $E_{c . m}$. at the collision event near the horizon. This is in contrast to the case discussed in [20], which is realized near the turning point of a usual particle with small $X_{H}$, and the turning point itself must be close to the horizon: the nearer it is, the larger $E_{c . m}$. is achieved.
} 
This can be turned around to give $L_{H}\left(x_{1}\right)$ and $L_{2}\left(x_{3}\right)$, but $x_{2} / x_{1}$ is fixed to metric function coefficients; in terms of $E(L)$ this is

$$
E=\omega_{H} L_{H}+\left(L_{2} \omega_{H}-L_{H} \omega_{2}\right) \xi^{2}+\ldots
$$

For other integer $s$ the procedure is analogous.

\section{ENERGY BOUNDS IN COLLISIONS WITH NEAR-CRITICAL PARTICLES}

We have seen in the previous section that as long as the azimuthal force is weak enough, critical particles exist and can be tuned via initial conditions. Then the BSW effect in its primary version [1] manifests itself. However, what if this is not the case and azimuthal force is e.g. separated from zero on the horizon? The condition for critical particles is that

$$
a^{(\phi)}=O(x)
$$

where

$$
x=\frac{\xi}{r_{H}}
$$

is the dimensionless radial coordinate. Suppose that instead

$$
a^{(\phi)}(x) \approx \frac{a_{0} x^{\lambda}}{r_{H}}, \quad \lambda<1
$$

so that (158) is violated, and we factored out the dimensional quantity $r_{H}^{-1}$, so that $a_{0}$ is dimensionless.

Does it mean that the BSW effect necessarily breaks down? Under no additional assumptions - yes. However, what if $a_{0}$ is small? Radiation reaction forces are usually considered very small (see e.g. [16], [17]). In that case, the question is how high $E_{c . m}$. can be achieved for the given small $a_{0}$ ?

Let us reformulate the condition that is satisfied (160) via another small parameter:

$$
a^{(\phi)}(x) \approx r_{H}^{-1} x^{\lambda} x_{m}^{1-\lambda}
$$


where

$$
x_{m}=a_{0}^{\frac{1}{1-\lambda}} \ll 1
$$

Then

$$
a^{(\phi)}\left(x_{m}\right) \approx r_{H}^{-1} x_{m}
$$

and for all $x \gtrsim x_{m}$ the necessary condition for acceleration (158) is effectively obeyed.

But then for $\xi \gtrsim r_{H} x_{m}$ the trajectory of a particle can be effectively tuned to be critical, (or sub-critical, for the chosen $p$ ), at will. At the near-horizon end of this region, i.e. at $\xi_{m} \sim r_{H} x_{m}$, the Lorentz factor with a usual particle with $p_{2}=0$ will behave as described (44) and can grow very large. Thus, for $p=1$ (the particle is tuned to be critical) from (42) we get

$$
\gamma_{12}^{(\max )} \approx \gamma_{12}\left(x_{m}\right) \approx \frac{C_{1} \alpha_{2}}{r_{H}} \cdot a_{0}^{-\frac{1}{1-\lambda}}
$$

We see that as long as the amplitude $a_{0}$ of the azimuthal force acting on the particle is small enough, the BSW effect survives almost any kind of perturbation: one only has to calculate accurately the corresponding tuning parameters for the effectively critical trajectories.

\section{KINEMATIC RESTRICTIONS ON CRITICAL PARTICLES AND TWO TYPES OF THE BSW EFFECT}

In the preceding Section, it was assumed that collision occurred not exactly on the horizon but at some coordinate distance from it, its scale being tied to the amplitude of azimuthal acceleration, which is supposed to be small. The force, being too large, prevents the critical particle from approaching the horizon. In this sense, the reason of it is dynamic. Meanwhile, even if the external force is small enough or absent at all, pure kinematic factors can also create an obstacle for reaching the horizon.

Let us remind the situation with geodesic particles. If the horizon is nonextremal, the critical particle cannot reach the horizon at all. Nonetheless, it was demonstrated in [7] for the Kerr metric and in [8] for generic dirty axially symmetric black holes, that $E_{c . m}$. can be made as large as one likes provided (i) the critical particle is replaced with a slightly noncritical one, (ii) the coordinate distance between the point of collision and horizon is adjusted to the small deviation of the particle's parameters from the values corresponding 
to the critical case.

Now, we are dealing with an extremal horizon but there is a special situation when $X \sim \xi^{p}$ with $p>1$ ("supercritical" particle). Such a particle cannot reach the horizon (in this sense it is similar to the case of the nonextremal horizon).

Then, instead of taking a critical particle, we can choose a usual one with sufficiently small $X_{H}$. More precisely, let us consider expansion for $X$ of the form

$$
X=X_{H}+\alpha_{s} \xi^{s}\left(1+x_{1} \xi+\ldots\right), \quad p>1 .
$$

Then, we look for the region in which both terms in $Z(7)$ are of the same order of magnitude. This is achieved at $\xi \approx \xi_{c} \sim r_{H} X_{H}$. Then we can neglect the correction in (165), so that

$$
Z\left(\xi_{c}\right) \sim X_{H} \sim \xi_{c} \sim N\left(\xi_{c}\right)
$$

and therefore (27) implies

$$
\gamma_{c . m .}^{(\max )} \approx \gamma_{c . m .}\left(\xi_{c}\right) \sim N^{-1}\left(\xi_{c}\right) \sim \xi_{c}^{-1} .
$$

Thus one can distinguish between two main types of the BSW effect: BSW 1, in which the critical particle can approach the horizon, so that the horizon limit can be taken, and BSW 2, for which the critical particle does not reach the horizon. We see that, in general, the presence of the external force is compatible with both types of the BSW effect ${ }^{10}$.

It is worth noting that even in the absence of force, the expansion for $X$ can take the form (165), if the linear terms cancel each other. Say, this happens for solutions near the so-called ultraextremal horizon $\left(N^{2} \sim\left(r-r_{H}\right)^{3}\right)$ in special "exotic" metrics described in Sec. IV B. 5 of [13], for which $\left.\partial_{r} \omega\right|_{H}=\left.\partial_{r}^{2} \omega\right|_{H}=0$, and thus $s$ can be equal to 2 or 3 . Correspondingly, the BSW-2 effect can be realized near such horizons.

${ }^{10}$ Another, more detailed classification of trajectories and corresponding types of the BSW effect can be found in Sec. IV of [16] for the Kerr metric and in [21] for general dirty rotating axially symmetric black holes. 


\section{CONCLUSION}

In general, three main circumstances were considered as the factors which were expected to restrict the indefinite growth of $E_{\text {c.m. }}$. and thus create obstacles to the manifestation of the BSW effect. These are (i) self-gravitation, (ii) deviation of a black hole from extremality [3], and (iii) the force due to backreaction of gravitational or electromagnetic radiation. As far as self-gravitation is concerned, it was shown in [18] that for collisions of massive shells, either the BSW effect does not occur or it occurs but in the region inaccessible by a remote observer. However, in this case the shell does not approach the horizon from the viewpoint of an external observer. As there is no horizon, there is no BSW effect. Factor (ii) was analyzed in [7] for the Kerr metric where it was shown that for nonextremal black holes the BSW effect does exist (this conclusion was generalized in [8] to generic dirty axially symmetric black holes).

And, in the present work, we showed for extremal horizons that the BSW effect is compatible with a nonzero force under rather general assumptions: the radial force should be finite and the azimuthal force should tend to zero not too slowly. In terms of energy and angular momentum, the kinematic condition for the realization of the BSW effect is the same as for geodesic particles: $E=\omega_{H} L$. In this sense, this condition by itself survives the action of the force (see also Sec. V of [6] where, however, another physical situation was considered - near-circular orbits around near-extremal black holes). Our approach is model-independent and is based on general properties of the horizons.

For the finite radial component of the force and the azimuthal one which tends to zero near the horizon as $r-r_{H}$, the BSW effect still exists. Otherwise, the effect is formally absent. The reason for the restriction on the azimuthal force seems to be clear: if azimuthal force was too large or did not tend to zero, in the infinite proper time that it takes for a critical particle to reach the horizon this force would accelerate the particle to infinite values of angular momentum. Obviously, one would not expect a force dissipative by nature, such as radiation reaction, to have such an effect. So, this only seeming restriction should be always obeyed. Even if it is not (so critical trajectories are absent), but the amplitude of the azimuthal force is small, the restrictions on $E_{c . m}$. are shown to be inessential, and one can still attain very high energies.

To summarize, the BSW effect turned out to be more viable than one could expect. 
The present work confirmed that the BSW effect relies on two main properties: (i) the presence of the horizon and (ii) the existence of special types of trajectories. Thus it has geometric nature and reflects general features of black holes irrespective of the details of the system. Concrete realization of the BSW effect certainly depends on particular properties of a system but near the horizon these properties manifest themselves in a universal way. We see that although dissipative forces in flat spacetime generically bound the values of energy peaks from above, in the strong gravitational field regime near the horizon the geometry dominates over the influence of dissipative forces on the system.

The present results refer to extremal horizons only. The nonextremal case and, especially, motion on circular orbits around near-extremal black holes, so important in astrophysical context, require separate treatment. Generalization to non-equatorial motion is also necessary. This will be done elsewhere.

[1] M. Banados, J. Silk, S.M. West, Phys. Rev. Lett. 103, 111102 (2009) [arXiv:0909.0169].

[2] E. Berti, V. Cardoso, L. Gualtieri, F. Pretorius, U. Sperhake, Phys. Rev. Lett. 103, 239001 (2009) [arXiv:0911.2243].

[3] T. Jacobson, T.P. Sotiriou, Phys. Rev. Lett. 104, 021101 (2010) [arXiv:0911.3363].

[4] V.P. Frolov, Phys. Rev. D 85, 024020 (2012) [arXiv:1110.6274].

[5] O.B. Zaslavskii, Gravitation and Cosmology 18, 139 (2012) [arXiv:1107.3964].

[6] T. Harada and M. Kimura, Phys. Rev. D 84124032 (2011) [arXiv:gr-qc/1109.6722].

[7] A.A. Grib and Yu.V. Pavlov, Pis'ma v ZhETF 92, 147 (2010) [JETP Letters 92, 125 (2010)].

[8] O.B. Zaslavskii, Phys. Rev. D 82083004 (2010) [arXiv:1007.3678].

[9] M. Bejger, T. Piran, M. Abramowicz, and F. Hå kanson, Phys. Rev. Lett. 109, 121101 (2012) [arXiv:1205.4350]

[10] T. Harada, H. Nemoto, and U. Miyamoto, Phys. Rev. D 86, 024-027 (2012) [arXiv:1205.7088]

[11] O.B. Zaslavskii, Phys. Rev. D 86, 084030 (2012) [arXiv:1205.4410]

[12] K.A. Bronnikov, S.G. Rubin, Black holes, cosmology and extra dimensions (World Scientific Publishing Company, 2012).

[13] I.V. Tanatarov, O.B. Zaslavskii, Phys. Rev. D 86, 044019 (2012) [arXiv:gr-qc/1206.2580].

[14] J. M. Bardeen, W. H. Press, and S. A. Teukolsky, Astrophys. J. 178, 347 (1972). 
[15] S. Caroll, Spacetime and geometry: an introduction to General Relativity (Addison-Wesley, 2004).

[16] T. Harada and M. Kimura, Phys. Rev. D 83 (2011) 084041 [arXiv:1102.3316].

[17] M. Kesden, Phys. Rev. D 83, 104011 (2011) [arXiv:1101.3749].

[18] M. Kimura, Ken-ichi Nakao and H. Tagoshi, Phys. Rev. D 83, 044013 (2011), [arXiv:1010.5438].

[19] O.B. Zaslavskii, Pis'ma ZhETF 92, 635 (2010) (JETP Letters 92, 571 (2010)), [arXiv:1007.4598].

[20] A.A. Grib and Yu.V. Pavlov, Astroparticle Physics 34, 581 (2011) [arXiv:1001.0756].

[21] O.B. Zaslavskii, JHEP, 2012, 32 (2012) [arXiv:1209.4987]. 